\title{
Equivalence between two different field-dependent BRST formulations
}

\author{
Sudhaker Upadhyay $*$ \\ Department of Physics, Indian Institute of Technology Kanpur, Kanpur 208016, India \\ Bhabani Prasad Manda I \\ Department of Physics, Banaras Hindu University, Varanasi 221005, India
}

\begin{abstract}
Finite field-dependent BRST (FFBRST) transformations were constructed by integrating infinitesimal BRST transformation in a closed form. Such a generalized transformations have been extended in various branch of physics and found many applications. Recently BRST transformation has also been generalized with same goal and motivation in slightly different manner. In this work we have shown that the later formulation is conceptually equivalent to the earlier formulation. We justify our claim by producing the same result of later formulation using earlier FFBRST formulation.
\end{abstract}

\section{INTRODUCTION}

The BRST symmetry transformation is a fundamental tool for the study of gauge theories [1, 2]. This symmetry guarantees the quantization, renormalizability, unitarity and other aspects of Yang-Mills (YM) theories [3 [5]. The derivation of Slavnov-Taylor identities [6, 7] in YM theory utilize the BRST symmetry transformation. In Hamiltonian formulation, the invariance of the theory under the gauge transformation of variables generated by the constraints is replaced by the invariance under the transformations generated by the Batalin-Fradkin-Vilkovisky (BFV)-BRST charge [3]. Action and measure of the related path integrals should be invariant under the BFV-BRST transformations.

The concept of FFBRST transformation was first introduced by Joglekar and Mandal in 1995 [8] in which they explicitly show that the Jacobian of path integral measure could be written exponential of some local functional of fields. This local functional of fields can be adjusted to a desirable form by constructing the finite field-dependent parameter appropriately. Due to this interesting result the FFBRST transformation is capable to relate the generating functionals of different effective theories. After this seminal work the FFBRST transformation have found great applicability in diverse area of gauge theories $[9-26]$. For instance, a correct prescription for poles in the gauge field propagators in noncovariant gauges has been derived by connecting covariant and noncovariant gauges for YM theory through FFBRST transformation [13]. The long outstanding problem of divergent energy integrals in Coulomb gauge was also settled by FFBRST transformation [10]. The YM theory in the non-perturbative infrared region is plagued by the Gribov ambiguity 27]. This ambiguity has been fixed by proposing the Gribov-Zwanziger theory [28] which restricts the domain of integration to the boundary called as Gribov first horizon. The FFBRST transformation has also played a crucial role in connecting the YM theory to Gribov-Zwanziger theory [29, 30]. The connections of the linear and the non-linear gauges for the perturbative theory of quantum gravity [21] and for Bagger-Lambert-Gustavsson (BLG) theory [23] at both classical and quantum level have been studied recently via FFBRST technique. Furthermore, the gauge-fixing and ghost terms corresponding to Landau as well as maximal Abelian gauge for the ChoFaddeev-Niemi (CFN) decomposed theory [31 33], which enables us to explain and understand some of the low-energy phenomena by separating the topological defects in a gauge-invariant manner, appear naturally within FFBRST formulation [19]. Moreover, the gaugeon formulation has also been justified

\footnotetext{
*Electronic address: sudhakerupadhyay@gmail.com
}

${ }^{\dagger}$ Electronic address: bhabani.mandal@gmail.com 
through FFBRST transformations [15, 20].

Recently, Lavrov and Lechtenfeld have worked on field-dependent BRST transformation [34] exactly similar to FFBRST formulation of Ref. [8] in slightly different way to claim it as a new formulation. The purpose of this work is to point out that their formulation is not a new one but the something similar to one which has been done 20 years ago in [8]. In this paper we show the equivalence between the original FFBRST formulation developed by Joglekar and Mandal, and the field-dependent BRST formulation studied by Lavrov and Lechtenfeld. The field-dependent parameter in Ref. [8] is finite as the Green function of such quantity is calculated between a vacuum and a state with gauge and ghost field which is finite rather than infinitesimal. Jacobian for the path integral measure has been calculated explicitly in terms of the finite parameter of the BRST transformation [34]. We found that although the ways of calculating Jacobian are different these two approaches are producing same results. We justify our claim by producing the results of [34] through the FFBRST formulation. We show the connection between different $R_{\xi}$ gauges using FFBRST transformation.

The paper is organized as follows. We provide a brief discussion on FFBRST formulation in Sec. II. Then, in section III, we compare two different approaches of generalizing the BRST transformation. In the last section, we draw conclusion.

\section{FFBRST TRANSFORMATION}

In this section let us revise the celebrated FFBRST formulation which has been found the enormous applications in gauge theories. To achieve this goal, we first define the usual BRST transformation for a generic field $\phi$ written collectively as follows:

$$
\delta_{b} \phi=s_{b} \phi \delta \lambda=\mathcal{R}[\phi] \delta \lambda,
$$

where $\mathcal{R}[\phi]=s_{b} \phi$ denotes the Slavnov variation of $\phi$ and $\delta \lambda$ is an anticommuting global parameter of transformation. Now, we write the infinitesimal field-dependent transformation by making all the fields $\kappa$-dependent, where $\kappa(0 \leq \kappa \leq 1)$ is a continuous parameter, as follows

$$
\frac{d \phi(x, \kappa)}{d \kappa}=\mathcal{R}[\phi(x, \kappa)] \Theta^{\prime}[\phi(x, \kappa)],
$$

where the $\Theta^{\prime}[\phi(x, \kappa)]$ is an infinitesimal field-dependent parameter. Integrating the above infinitesimal field-dependent transformation from $\kappa=0$ to $\kappa=1$, we obtain the following FFBRST transformation $\left(\delta_{f}\right)$ :

$$
\delta_{f} \phi(x) \equiv \phi(x, \kappa=1)-\phi(x, \kappa=0)=\mathcal{R}[\phi(x)] \Theta[\phi(x)],
$$

where

$$
\Theta[\phi]=\Theta^{\prime}[\phi] \frac{\exp f[\phi]-1}{f[\phi]},
$$

is the finite field-dependent parameter and $f[\phi]$ is given by

$$
f[\phi]=\sum_{i} \int d^{4} x \frac{\delta \Theta^{\prime}[\phi]}{\delta \phi_{i}(x)} s_{b} \phi_{i}(x) .
$$

Now, it has been found that the FFBRST transformation having such field-dependent parameter leads a non-trivial Jacobian for the functional measure of the generating functional [8]. We provide a glimpse of computing the Jacobian of the path integral measure $(\mathcal{D} \phi)$ in the functional integral under FFBRST transformation. In this regard, the first step is to define the functional measure under the FFBRST transformation as follows [8]

$$
\mathcal{D} \phi^{\prime}=J(\kappa) \mathcal{D} \phi(\kappa) .
$$


It has been shown explicitly in [8] that within functional integral the Jacobian $J(\kappa)$ can be replaced as

$$
J(\kappa) \longmapsto e^{i S_{1}[\phi(x, \kappa)]},
$$

where $S_{1}[\Phi]$ is some local functional of fields, if and only if the condition

$$
\int \mathcal{D} \phi(x)\left[\frac{d}{d \kappa} \ln J(\kappa)-i \frac{d S_{1}[\phi(x, \kappa)]}{d \kappa}\right] \exp \left[i\left(S_{e f f}+S_{1}\right)\right]=0
$$

is satisfied. However, the local functional $S_{1}[\Phi]$ satisfies the following initial boundary condition

$$
S_{1}[\Phi]_{\kappa=0}=0 .
$$

The infinitesimal change in Jacobian written in (8) has the following explicit expression [8]

$$
\frac{d}{d \kappa} \ln J(\kappa)=-\int d^{4} y\left[ \pm \sum_{i} \mathcal{R}\left[\phi^{i}(y)\right] \frac{\partial \Theta^{\prime}[\phi(y, \kappa)]}{\partial \phi^{i}(y, \kappa)}\right],
$$

where we employ the positive $(+)$ sign for bosonic fields and however for fermionic fields we use the negative (-) sign. Hence the vacuum functional for a most general gauge theory defined by

$$
Z=\int \mathcal{D} \phi e^{i S_{e f f}}
$$

changes under FFBRST transformation due to Jacobian as

$$
Z\left(\equiv \int \mathcal{D} \phi e^{i S_{e f f}}\right) \stackrel{F F B R S T}{\longrightarrow} Z^{\prime}\left(\equiv \int \mathcal{D} \phi e^{i\left(S_{e f f}+S_{1}\right)}\right)
$$

where $S_{\text {eff }}$ is the effective action for this most general theory. By constructing an appropriate parameter $\Theta$, the expression for the functional $S_{1}$, which extends the effective action, can be manipulated according to the requirement of the theory.

\section{COMPARISON BETWEEN DIFFERENT FIELD-DEPENDENT BRST FORMULATIONS: THE YM THEORY}

Let us start the section by defining the vacuum functional for YM theory in $R_{\xi}$ gauge using the standard Faddeev-Popov method as follows

$$
Z_{\xi}=\int \mathcal{D} \phi e^{i S_{\xi}}
$$

where $\mathcal{D} \phi$ is the generic path integral measure written in terms of all fields involved in the theory and the effective action, $S_{\xi}$, comprised with classical and gauge-fixed parts is defined by

$$
S_{\xi}=\int d^{4} x\left[-\frac{1}{4} F^{a \mu \nu} F_{\mu \nu}^{a}+\frac{\xi}{2}\left(B^{a}\right)^{2}+B^{a} \partial^{\mu} A_{\mu}^{a}+\bar{C}^{a} \partial^{\mu} D_{\mu}^{a b} C^{b}\right] .
$$

The covariant derivative is expressed as follows: $D_{\mu}^{a b} \equiv \delta^{a b} \partial_{\mu}+g f^{a b c} A_{\mu}^{c}$, where $g$ denotes the coupling constant.

The usual BRST transformations under which the effective action (14) remains invariant are given by

$$
\begin{aligned}
\delta_{b} A_{\mu}^{a} & =D_{\mu}^{a b} C^{b} \delta \lambda, \\
\delta_{b} C^{a} & =-\frac{g}{2} f^{a b c} C^{b} C^{c} \delta \lambda, \\
\delta_{b} \bar{C}^{a} & =B^{a} \delta \lambda, \\
\delta_{b} B^{a} & =0 .
\end{aligned}
$$


Using the above BRST transformations the effective action can also be expressed in terms of gauge-fixing fermion $(\psi)$ as follows:

$$
S_{\xi}=\int d^{4} x\left[-\frac{1}{4} F^{a \mu \nu} F_{\mu \nu}^{a}+s_{b} \psi\right],
$$

where the gauge-fixing fermion has following expression:

$$
\psi=\bar{C}^{a}\left(\partial^{\mu} A_{\mu}^{a}+\frac{\xi}{2} B^{a}\right) .
$$

Following the procedure outlined in Sec. III, the FFBRST transformations for YM theory are constructed by

$$
\begin{aligned}
\delta_{f} A_{\mu}^{a} & =D_{\mu}^{a b} C^{b} \Theta[\phi], \\
\delta_{f} C^{a} & =-\frac{g}{2} f^{a b c} C^{b} C^{c} \Theta[\phi], \\
\delta_{f} \bar{C}^{a} & =B^{a} \Theta[\phi], \\
\delta_{f} B^{a} & =0,
\end{aligned}
$$

where $\Theta[\phi]$ is an arbitrary finite field-dependent parameter. For instance, we choose an specific $\Theta[\phi]$ obtained from the following infinitesimal field-dependent parameter using relation (4)

$$
\Theta^{\prime}[\phi]=\int d^{4} y\left[\bar{C}^{a} B^{a}\left(B^{2}\right)^{-1}\left(\frac{\delta \xi}{2 i} B^{2}\right)\right] .
$$

The finite field-dependent parameter is calculated by

$$
\Theta[\phi]=\int d^{4} y\left[\bar{C}^{a} B^{a}\left(B^{2}\right)^{-1}\left(\exp \left\{\frac{\delta \xi}{2 i} B^{2}\right\}-1\right)\right],
$$

which coincides with the parameter given in 34].

Exploiting the relations (10) and (19), we compute the change in Jacobian as follows:

$$
\frac{d}{d \kappa} \ln J(\kappa)=-i \int d^{4} x\left[\frac{\delta \xi}{2} B^{2}\right],
$$

An ansatz for the (arbitrary) local functional $S_{1}[\phi]$ which appears in the expression (exponent) of the Jacobian (7) as

$$
S_{1}[\phi]=\int d^{4} x\left[\chi(\kappa) B^{2}\right],
$$

where $\chi(\kappa)$ is an arbitrary constant parameter constrained by

$$
\chi(\kappa=0)=0,
$$

so that the requirement (9) holds. Now, the change in $S_{1}$ with respect to $\kappa$ can easily be calculated by utilizing the relation (2) as follows:

$$
\frac{d S_{1}}{d \kappa}=\int d^{4} x\left[\frac{d \chi}{d \kappa} B^{2}\right] .
$$

The existence of the functional $S_{1}$ is valid when it satisfies the essential requirement given in (8) along with (21) and (24). This leads to the following exactly solvable linear differential equation:

$$
\frac{d \chi}{d \kappa}+\frac{\delta \xi}{2}=0
$$


The unique solution of the above equation satisfying the initial boundary condition (9) is given by

$$
\chi(\kappa)=-\frac{\delta \xi}{2} \kappa .
$$

Having this specific value of $\chi$ the expression of $S_{1}$ at $\kappa=1$ reads

$$
\left.S_{1}[\phi]\right|_{\kappa=1}=-\int d^{4} x\left[\frac{\delta \xi}{2} B^{2}\right] .
$$

This implies that the effective action within functional integral under the action of FFBRST transformation changes to

$$
\begin{aligned}
S_{\xi}+\left.S_{1}[\phi]\right|_{\kappa=1} & =\int d^{4} x\left[-\frac{1}{4} F^{a \mu \nu} F_{\mu \nu}^{a}+\frac{(\xi-\delta \xi)}{2}\left(B^{a}\right)^{2}+B^{a} \partial^{\mu} A_{\mu}^{a}+\bar{C}^{a} \partial^{\mu} D_{\mu}^{a b} C^{b}\right], \\
& =: S_{\xi-\delta \xi},
\end{aligned}
$$

which is nothing but the effective YM action in $R_{\xi-\delta \xi}$ gauge. However, for $\delta \xi=\xi$, the connection between Landau and $R_{\xi}$ can easily be established. Therefore, FFBRST transformation with specific parameter mentioned in (19) connects the $R_{\xi}$ and $R_{\xi-\delta \xi}$ gauges of the YM theory. In summery we see that

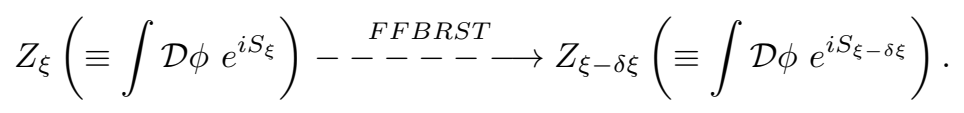

Hence, we have obtained exactly same results of Ref. [34] using the formulation of Ref. [8] which was developed long ago. These results justifies the equivalence between the two field-dependent BRST approaches, namely, the original FFBRST approach [8] and the recently developed field-dependent BRST approach advocated in [34].

\section{CONCLUSIONS}

The finite field-dependent BRST (FFBRST) transformation was developed long ago [8] and has been found great importance in the different context of gauge field theories. Recently, a new method has been explored to study the field-dependent BRST transformation [34]. Although being presented differently we have found that these two approaches are exactly same. The novelty of these symmetry transformations are: they lead a non-trivial Jacobian for the functional measure under change of variables and capable of connecting the generating functional of different effective theories. In this paper, we have calculated the Jacobian for path integral measure using former FFBRST method which are found same to that of calculated by the later approach. Further, we have shown that the FFBRST transformation amounts a precise change in the functional integral of YM theory as shown in Ref. [34]. Our study establishes the equivalence between the two approaches of FFBRST transformations.

[1] C. Becchi, A. Rouet and R. Stora, Annals Phys. 98287 (1974).

[2] I. V. Tyutin, LEBEDEV-75-39 (1975).

[3] M. Henneaux and C. Teitelboim, Quantization of gauge systems, Princeton, USA: Univ. Press (1992).

[4] S. Weinberg, The quantum theory of fields, Vol-II: Modern applications, Cambridge, UK Univ. Press (1996).

[5] M. Chaichian and N. F. Nelipa, Introduction to Gauge Field Theories, Springer Berlin Heidelberg, (2012).

[6] A. A. Slavnov, Theor. Math. Phys. 10, 99 (1972).

[7] J. C. Taylor, Nucl. Phys. B 33, 436 (1971).

[8] S. D. Joglekar and B. P. Mandal, Phys. Rev. D 51, 1919 (1995).

[9] B. P. Mandal, S. K. Rai and S. Upadhyay, EPL 92, 21001 (2010). 
[10] S. D. Joglekar and B. P. Mandal, Int. J. Mod. Phys. A 17, 1279 (2002).

[11] R. Banerjee and B. P. Mandal, Phys. Lett. B 488, 27 (2000).

[12] S. Upadhyay, S. K. Rai and B. P. Mandal, J. Math. Phys. 52, 022301 (2011).

[13] S. D. Joglekar and A. Misra, Int. J. Mod. Phys. A 15, 1453 (2000).

[14] S. Upadhyay and B. P. Mandal, Eur. Phys. J. C 72, 2065 (2012); Annals of Physics 327, 2885 (2012); Mod. Phys. Lett. A 25, 3347 (2010).

[15] S. Upadhyay and B. P. Mandal, Prog. Theor. Exp. Phys. 053B04, (2014).

[16] S. Upadhyay, M. K. Dwivedi and B. P. Mandal, Int. J. Mod. Phys. A 28, 1350033 (2013).

[17] S. Upadhyay and D. Das, Phys. Lett. B 733, 63 (2014).

[18] S. Upadhyay, EPL 104, 61001 (2013); EPL 105, 21001 (2014); Phys. Lett. B 740, 341 (2015); arXiv:1502.05217. arXiv:1503.04197.

[19] S. Upadhyay, Phys. Lett. B 727, 293 (2013).

[20] S. Upadhyay, Annals of Physics 344, 290 (2014).

[21] S. Upadhyay, Annals of Physics 340, 110 (2014).

[22] M. Faizal, S. Upadhyay and B. P. Mandal, Phys. Lett. B 738, 201 (2014); Int. J. Mod. Phys. A 30, 1550032 (2015).

[23] M. Faizal, B. P. Mandal and S. Upadhyay, Phys. Lett. B 721, 159 (2013).

[24] R. Banerjee, B. Paul and S. Upadhyay, Phys. Rev. D 88, 065019 (2013).

[25] R. Banerjee and S. Upadhyay, Phys. Lett. B 734, 369 (2014).

[26] S. K. Rai and B. P. Mandal, Int. J.Theor. Phys. 52, 3512 (2013).

[27] V. N. Gribov, Nucl. Phys. B 139, 1 (1978).

[28] D. Zwanziger, Nucl. Phys. B 323, 513 (1989).

[29] S. Upadhyay and B. P. Mandal, EPL 93, 31001 (2011).

[30] S. Upadhyay and B. P. Mandal, AIP Conf. Proc. 1444, 213 (2012).

[31] Y. M. Cho, Phys. Rev. D 21, 1080 (1980); Phys. Rev. D 23, 2415 (1981).

[32] Y. S. Duan and M. L. Ge, Sinica Sci. 11, 1072 (1979).

[33] L. Faddeev and A. J. Niemi, Phys. Rev. Lett. 82, 1624 (1999).

[34] P. M. Lavrov and O. Lechtenfeld, Phys. Lett. B 725, 382 (2013). 